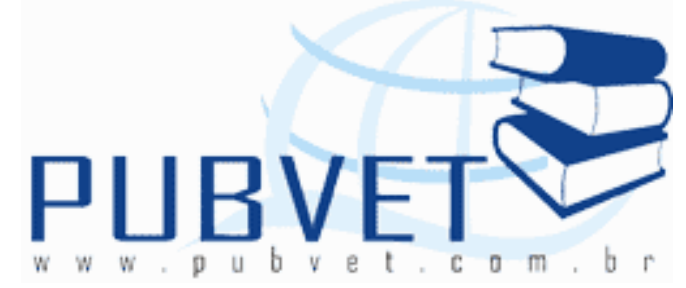

PUBVET, Publicações em Medicina Veterinária e Zootecnia.

\title{
Análise de esfregaço fecal para diagnóstico de megabactéria (Macrorhabdus ornithogaster) em aves domésticas e silvestres
}

\footnotetext{
Thiago Silva de Queiros ${ }^{1}$, Patricia Lourenço ${ }^{2}$ e Maria Carolina Gonçalves Pita ${ }^{3}$

${ }^{1}$ Graduando em Medicina Veterinária, Universidade Guarulhos.

${ }^{2}$ Responsável pelo laboratório de análises clínicas, Universidade Guarulhos.

3 Docente das disciplinas Ornitopatologia e Manejo de Animais Silvestres, Medicina Veterinária, Universidade Guarulhos.
}

\section{Resumo}

A megabacteriose, também chamada de "Síndrome Light Going" é causada por um microorganismo grande, em forma de bastão, Gram-positivo, encontrado na flora normal do proventrículo das aves, como: periquito australiano (Melopsittacus undulatus), canário (Serinus canarius), ema (Rhea americana), agapornis (Agapornis roseicollis), tucano (família Ramphastidae), avestruz (Struthio camelus). Este trabalho tem como objetivo relatar a ocorrência de Macrorhabdus ornithogaster, agente causador das megabacterioses, em agapornis (Agapornis roseicollis), canários (Serinus canarius) e periquitos australianos (Melopsittacus undulatus) de um criadouro particular do Estado de São Paulo/SP.

Palavras-chave: Macrorhabdus ornithogaster, megabactéria, agapornis, canários, periquito australiano. 


\title{
Analysis of fecal smears for diagnosis of megabactéria (Macrorhabdus ornithogaster) in domestic and wild birds
}

\begin{abstract}
The megabacteriosis, also called "Síndrome Light Going" caused for large rod shaped organism, Gram-positive, encountered in the normal flora of the proventricle of birds, such as budgerigars (Melopsittacus undulatus), canaries (Serinus canarius), rhea (Rhea americana), lovebirds (Agapornis roseicollis), toucan (Ramphastidae family), ostrich (Struthio camelus). This paper reports the occurrence of an Macrorhabdus ornithogaster, agent originator megabacteriosis, in lovebirds (Agapornis roseicollis), canaries (Serinus canarius) e budgerigars (Melopsittacus undulatus) of a particular property of the State of São Paulo / SP.
\end{abstract}

Keywords: Macrorhabdus ornithogaster, megabacteria, lovebirds, canaries, budgerigars.

\section{Introdução}

A megabacteriose é uma enfermidade caracterizada por emagrecimento progressivo, regurgitação, alterações nas fezes, apatia e morte (WERTHER et al., 2000). Também chamada de "Síndrome Light Going", é causada por um fungo que afeta muitas espécies de aves, tendo sido associada a uma condição crônica sintomática ou assintomática (MOORE et al., 2001; GERLACH, 2001).

A afecção foi relatada em alguns países e em diversas espécies de aves, domésticas e silvestres, tais como: periquito australiano (Melopsittacus undulatus), canário (Serinus canarius), ema (Rhea americana), agapornis (Agapornis roseicollis), cacatua (Cacatua sp), calopsita ou gutarra (Nymphicus hollandicus), mandarim (Taeniopygia guttata), tucano (família Ramphastidae), avestruz (Struthio camelus), pomba (família Columbidae), peru (Meleagrins gallopavo) e codorna-japonesa (Coturnix coturnix) (WERTHER et al., 2000; MARTINS et al., 2006; QUEIROS et al., 2011). 
De acordo com os estudos de Son et al. (2004) e Gerlach (2001), o agente foi caracterizado como uma estrutura bacilar grande que mede cerca de 1.0 a $5.0 \mu \mathrm{m}$ de diâmetro e 20 a $90 \mu \mathrm{m}$ de comprimento, Gram positivo, PAS (Periodic Acid Schiff) positivo. Mais recentemente Tomaszewski et al. (2003), realizaram a análise filogenética da megabactéria das aves, classificando o micro-organismo como um fungo ascomiceto anamórfico, denominado Macrorhabdus ornithogaster.

Segundo Munhoz et al. (2008), Macrorhabdus ornithogaster é um agente oportunista que se torna patogênico em situações de imunossupressão, manifestando sua forma clínica. A patogenia não é totalmente esclarecida, sabe-se que a megabactéria coloniza as porções inferiores do ventrículo, mais especificamente a membrana coilina, penetrando profundamente nesta, com isso ocorre hipersecreção das glândulas mucosas e espessamento da parede do ventrículo, associado a pequenas hemorragias (WERTHER et al., 2000; GUIMARÃES, 2006; GIOIA-DI CHIACCHIO et al., 2009).

A principal fonte de infecção da megabactéria são os portadores assintomáticos (MUNHOZ et al., 2008). De acordo com Moore et al. (2001) a alimentação através da regurgitação e a contaminação oro-fecal são as formas mais comuns de contaminação.

Martins et al. (2006), denotaram que condições de manejo incorreto, como alojamento conjunto de espécies diferentes, condições sanitárias inadequadas e precária biossegurança, aumentam as chances de transmissão do agente. De acordo com os estudos de Moore et al. (2001), a transmissão vertical da megabacteriose não ocorre.

Os métodos para deteç̧ão do Macrorhabdus ornithogaster incluem histórico clínico, anamnese, sinais clínicos, microscopia direta de impressão fresca da mucosa do ventrículo ou proventrículo, cultura de amostras de mucosa ventricular ou proventricular, exame histopatológico, necropsia e 
presença do agente nas fezes (MARTINS et al., 2006; DORRESTEIN, 2010; FERRONATO et al., 2011).

O objetivo do presente estudo foi realizar a análise de esfregaço fecal para pesquisa e diagnóstico de megabactéria (Macrorhabdus ornithogaster) em aves domésticas e silvestres.

\section{Material e Método}

\section{Formação dos Grupos Experimentais}

Para realização do projeto foram utilizadas 60 aves domésticas e silvestres, alojadas de maneira aleatória em cativeiro particular.

As aves foram submetidas a exames clínicos e as amostras de fezes foram colhidas para exame de esfregaço de fezes.

\section{Avaliação Clínica dos Animais}

O exame clínico dos animais foi realizado no momento da coleta de amostras de fezes, observando possíveis alterações no quadro clínico, como sinais de diarreia (fezes em volta da cauda, cloaca e membros inferiores) e alteração nas características comportamentais (apatia, stress e agressividade).

No exame físico foram avaliados pele e penas (cor, aspecto, apteria, presença de ectoparasitas, lesões nodulares), escore corporal, exame abdominal através de palpação, sendo observado possível aumento de volume do fígado e distensão das alças intestinais.

\section{Colheita e Avaliação das Amostras}

Foram colhidas amostras de fezes recentes, livres de substratos ou fezes de outros animais. As fezes foram coletadas do fundo da gaiola individual, previamente protegido por plástico estéril, e armazenadas em um frasco coletor universal limpo de tampa com rosca, com refrigeração temporária. 
As amostras foram enviadas ao laboratório de análises clínicas da Universidade Guarulhos, onde foi realizado o esfregaço fecal, para pesquisa microscópica de megabactéria (Macrorhabdus ornithogaster) através da coloração de Gram e visualização em microscópio óptico comum com aumento de 1.000 vezes, assim como realizado nos estudos de Martins et al. (2006) e Werther et al. (2000).

\section{Resultados}

O diagnóstico de infecção por megabactéria através da análise de esfregaço fecal em microscópio óptico comum com aumento de 1.000 vezes foi positivo em 29 das 60 aves (Tabela 1) provenientes do criadouro.

Tabela 1: Resultado de análise de esfregaço fecal de aves provenientes de cativeiro.

\begin{tabular}{l|lcc}
\hline Nome Comum & Nome Científico & $\begin{array}{c}\text { No de Aves } \\
\text { Positivas }\end{array}$ & $\begin{array}{c}\text { No de Aves } \\
\text { Negativas }\end{array}$ \\
\hline Agapornis & Agapornis roseicollis & 05 & 09 \\
Canário & Serinus Canarius & 09 & 12 \\
Periquito australiano & Melopsittacus undulatus & 15 & 10 \\
\hline Total & & $\mathbf{2 9}$ aves & $\mathbf{3 1}$ aves \\
\hline
\end{tabular}

Mediante análise dos esfregaços de fezes verificou-se diversas estruturas (Figura 1) com formato bacilar e tamanho alongado, que variam de $20 \mu \mathrm{m}$ a $90 \mu \mathrm{m}$ de comprimento e $1.5 \mu \mathrm{m}$ a $3.0 \mu \mathrm{m}$ de diâmetro, Gram-positivas, condizentes com a descrição do Macrorhabdus ornithogaster feita por Scanlan \& Graham (1990), Gerlach (2001) e Hannafusa et al. (2007).

Os resultados obtidos nesse estudo apontaram a presença do agente em $48,3 \%$ das amostras analisadas, apresentando positividade baixa a acentuada e variando de duas $(++/ 4)$ a quatro $(++++/ 4)$ cruzes. 
Os agapornis (Agapornis roseicollis) apresentaram 35,7\% de animais positivos, já os canários (Serinus canárius) apresentaram $42,9 \%$ de positivos e os periquitos australianos (Melopsittacus undulatus) apresentaram $60 \%$ de animais positivos. Embora o agente tenha sido encontrado em um percentual elevado de aves, a severidade foi variável, caracterizada por quadros de manifestações discretas ou até mesmo assintomáticas, porém o criador relatou o óbito de dois canários, de recintos distintos, semanas antes da coleta das amostras.

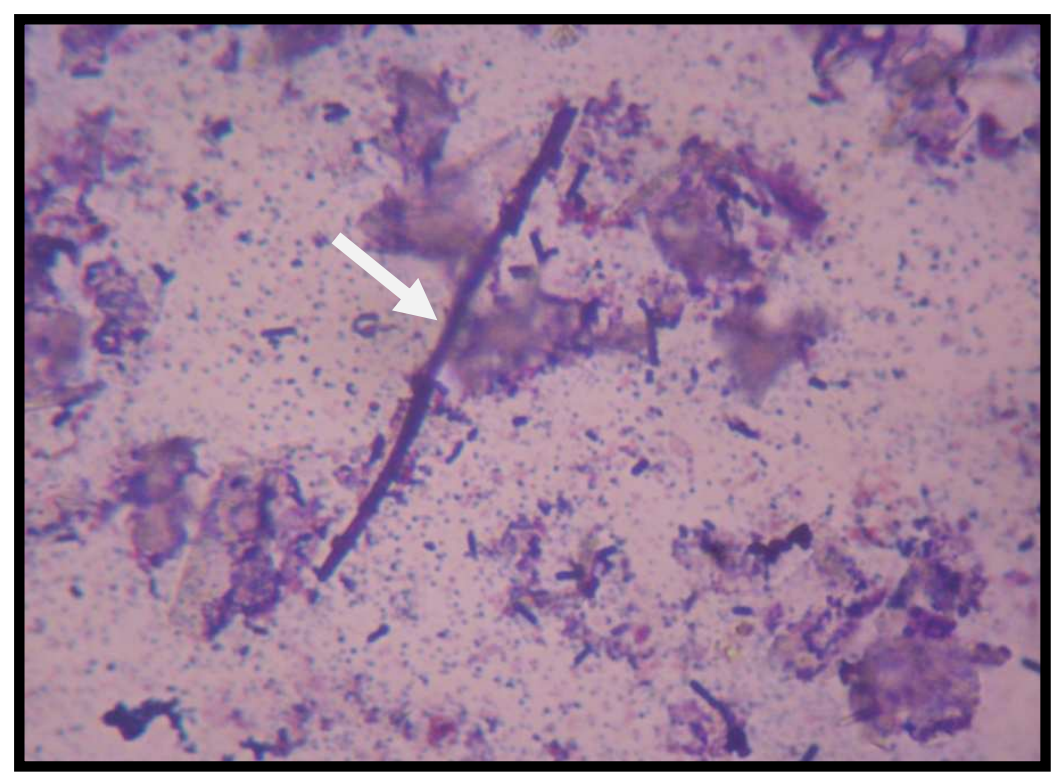

Figura 1: Megabactéria em esfregaço fecal, estrutura Gram-positiva de tamanho alongado. Análise realizada em microscópio óptico comum, em aumento de 1000 vezes. Fonte: Arquivo pessoal.

O criador relatou que as aves foram adquiridas, totalmente assintomáticas, de quatro criadouros distintos e acondicionadas de forma aleatória em seis gaiolas de aço inoxidável pertencentes ao cativeiro do local. Relatou também que entre um e dois dias $25 \%$ dos animais apresentaram alterações de comportamento e anorexia, caracterizando situações de imunossupressão, que pode provocar a proliferação do agente e a 
QUEIROS, T.S., LOURENÇO, P. e PITA, M.C.G. Análise de esfregaço fecal para diagnóstico de megabactéria (Macrorhabdus ornithogaster) em aves domésticas e silvestres. PUBVET, Londrina, V. 7, N. 13, Ed. 236, Art. 1559, Julho, 2013.

manifestação clínica da enfermidade, conforme denotaram Munhoz et al. (2008) e Ferronato et al. (2011).

\section{Discussão}

A megabacteriose é uma doença caracterizada por alta morbidade e baixa mortalidade que acomete uma grande variedade de aves (MARTINS et al., 2006). A gravidade dos sinais aumenta com a manifestação de quadros de imunossupressão ou associação com outros agentes, tais como os da coccidiose em tucanos, patógenos que causam diarreia em galinhas e codornas japonesas e da tricomoníase em pombos, aumentando a mortalidade que pode chegar até $100 \%$ do lote (SEGABINAZI et al., 2004; MARTINS et al., 2006; MUNHOZ et al., 2008).

Filippich \& Hendrikz (1998) diagnosticaram a enfermidade em uma série de espécies de aves, incluindo periquitos. Já Baker (1997), relatou em seus estudos que a presença de megabactéria é uma causa extremamente comum doença e morte em periquitos, alem disso, indica que $33 \%$ das aves acometidas não apresentam sinais ou lesões.

A megabactéria é um micro-organismo de distribuição mundial (MUNHOZ et al., 2008; FÁCCIO et al., 2010). No Brasil, Werther et al. (2000) observaram a presença de estruturas filiformes e Gram positivas, correspondentes com as estruturas observadas nesse estudo (Figuras 1 e 2), em $56 \%$ das aves necropsiadas no Hospital Veterinário da Universidade de São Paulo, no período de 1994 a 1997.

Apresenta sinais gastrointestinais graves como: diarreia, êmese, perda de peso progressiva, alimento mal digerido nas fezes, fezes aquosas e de cor verde-escura a marrom ou preta, e em casos mais graves fezes com sangue (WERTHER et al., 2000; DORRESTEIN, 2010; QUEIROS et al. 2011). 


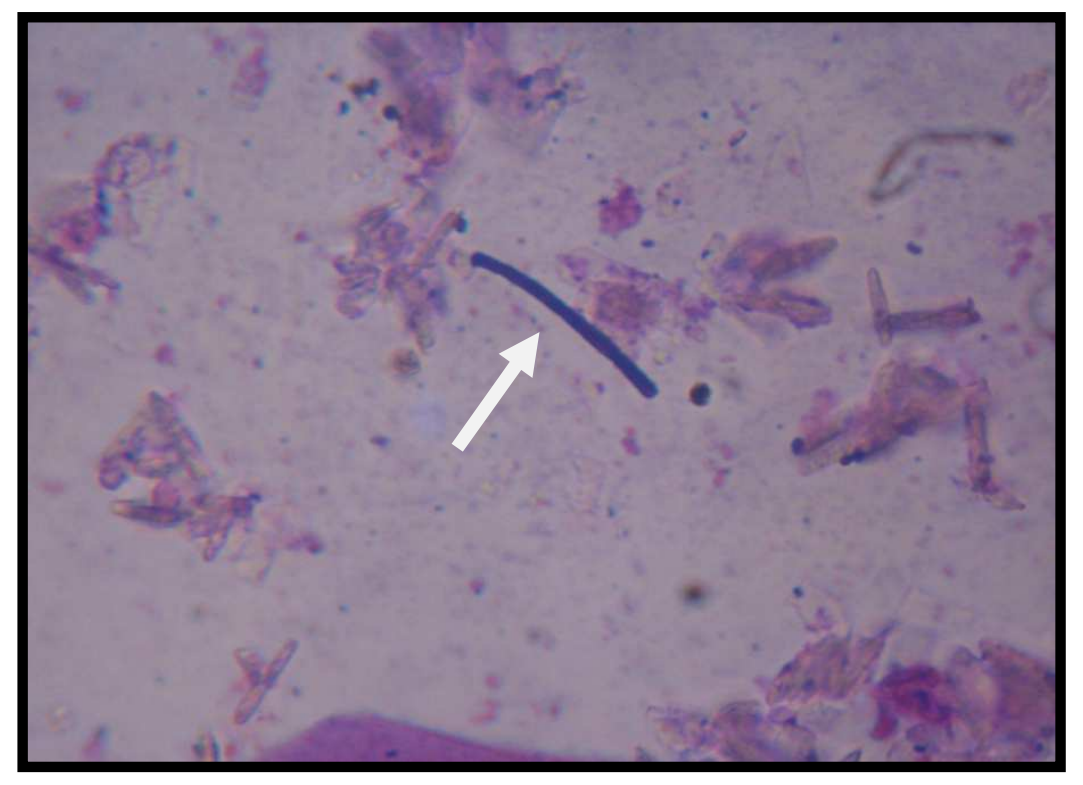

Figura 2: Esfregaço de fezes contendo Macrorhabdus ornithogaster, estrutura de formato bacilar e alongado, de tamanho variável, encontrado na flora bacteriana do trato digestivo. Fonte: Arquivo pessoal.

Os sinais clínicos incluem também crescimento irregular, depressão, anorexia, disfagia, fraqueza, letargia, problemas com empenamento, além de dramática atrofia dos músculos peitorais, acúmulo de fezes ao redor da cloaca, queda de postura, baixo ganho de peso e caquexia (BAKER, 1997; WERTHER et al., 2000; MOORE et al., 2001).

Um grupo correspondente a $23,3 \%$ do número total de animais apresentou sinais descritos por Martins et al. (2006) e Ferronato et. al. (2011) como característicos com o quadro de megabacteriose, tais como diarreia, anorexia, disfagia, perda de peso progressiva, fraqueza e depressão. Já o outro grupo de animais, correspondente aos outros $76,7 \%$, não apresentava qualquer sinal aparente. Esses dados indicam que a megabactéria pode ou não ser um micro-organismo da flora gastrointestinal das aves como já foi sugerido em outros estudos (QUEIROS et al., 2011).

Nesse estudo, pode-se considerar que uma das causas do número elevado de aves com diagnóstico positivo de megabactéria ocorreu por conta das condições de manejo incorreto, como alojamento conjunto de espécies 
QUEIROS, T.S., LOURENÇO, P. e PITA, M.C.G. Análise de esfregaço fecal para diagnóstico de megabactéria (Macrorhabdus ornithogaster) em aves domésticas e silvestres. PUBVET, Londrina, V. 7, N. 13, Ed. 236, Art. 1559, Julho, 2013.

diferentes, condições sanitárias inadequadas e precária biossegurança, que aumentam as chances de transmissão do agente, como foi descrito por Martins et al. (2006).

\section{Conclusão}

Através da análise dos esfregaços de fezes, comprovamos a presença de megabactéria (Macrorhabdus ornithogaster) em agapornis, canários e periquitos, mantidos em cativeiro.

Em nossos estudos, podemos concluir que a técnica de coloração pelo método de Gram foi eficiente na pesquisa de megabactéria, possibilitando o diagnóstico da enfermidade, mesmo em animais assintomáticos.

Vale ressaltar que ainda são necessários novos estudos, pois ainda não foi bem definido se o micro-organismo faz parte da flora gastrointestinal das aves.

\section{Referências Bibliográficas}

BAKER, J. R. Megabacteria in diseased and healthy budgerigars. Veterinary Record, v. 140, p. 627, 1997.

DORRESTEIN, G. M.; Passeriformes. In: TULLY, T. N.; DORRESTEIN, G. M.; JONES, A. K. Clínica de Aves, Rio de Janeiro: Elsevier, cp. 8, p.117, 2010.

FÁCCIO, C.; LUZ, P.G.; NUNES, C. F.; MEDEIROS, D. M.; SALLIS, S. E.; ALBANO, A. P. et al. Ocorrência de Macrorhabdus ornithogaster em aves silvestres e ornamentais da região Sul do Rio Grande do Sul. Anais XIX CIC - XII ENPOS - II Mostra Científica, 2010.

GERLACH, H. Megabacteriosis. Seminars in Avian and Exotic Pet Medicine, v. 10, n. 1, p. $12-19,2001$.

GUIMARÃES, M. B. Passeriformes (Pássaro, Canário, Saíra, Gralha). In: CUBAS, Z. S.; SILVA, J. C. R.; CATÃO-DIAS, J. L. Tratado de Animais Selvagens, São Paulo: Roca, cp. 22, p. 324$337,2006$.

HANNAFUSA, Y.; BRADLEY, A.; TOMASZEWSKI, E. E.; LIBAL, M. C.; PHALEN, D. N. Growth and metabolic characterization of Macrorhabdus ornithogaster. Journal of Veterinary Diagnostic Investigation, v. 19, p. 256-265, 2007.

FERronato, P. H.; lovato, M.; CEOlin, L. V.; CORRÊA, I. M. O.; MACEdo, A.; SCHNEIDERS, G. H. Casuística de canários atendidos pelo LCDPA com megabacteriose entre 2009 e 2011. Anais 26a Jornada Acadêmica Integrada, 2011. 
FILIPPICH, L. J. \& HENDRIKZ, J. K. Prevalence of megabacteria in budgerigar colonies. Australian Veterinary Journal, v. 76, p. 92-95, 1998.

GIOIA-DI CHIACCHIO, R. M.; PRIOSTE, F. S.; KNOBL, T.; SAINDENBERG, A. B. S. Megabacteriose em canários (Serinus canaria). Anais do Congresso Abravas, p.13-17, 2009.

MARTINS, N. R. S.; HORTA, A. M.; SIQUEIRA, A. M.; LOPES, S. Q.; RESENDE, J. S.; JORGE, M. A. et al. Macrorhabdus ornithogaster in ostrich, rhea, canary, zebra finch, free range chicken, turkey, guinea-fowl, columbina pigeon, toucan, chuckar partridge and experimental infection in chicken, japanese quail and mice. Arquivo Brasileiro de Medicina Veterinária e Zootecnia, v. 3, p. 291-298, 2006.

MOORE, R. P.; SNOWDEN, K. F. \& PHAKEN, D. N. A method of preventing transmission of socalled 'megabacteria' in budgerigars (Melopsittacus undulates). Journal of Avian Medicine and Surgery, v. 15, p. 283-287, 2001.

MUNHOZ, L. S.; FINGER, P. F.; SIEDLER, B. S.; FISCHER, G.; HUBNER S. O.; SALLIS, S. E. et al. Presença de Macrorhabdus ornithogaster em canários belga (Serinus canarius) oriundos da cidade de Pelotas - Rio Grande do Sul. Congresso Brasileiro de Medicina Veterinária, v. 35, p. 491-492, 2008.

QUEIROS, T. S. CARVALHO, P. R. e PITA, M. C. G. Megabacteriose: Macrorhabdus ornithogaster em Aves - Revisão. PUBVET, Londrina, v. 5, n. 13, Ed. 160, Art. 1080, 2011.

SCANLAN, C. M. \& GRAHAM, D. L. Characterization of a Gram-positive bacterium from proventriculus of budgerigars (Melopsittacus undulates). Avian Diseases, v. 34, p. 779-786, 1990.

SEGABINAZI, S. D.; FLÔRES, M. L.; KOMMERS, G. D.; BARCELOS, A. S.; VEIT, D. C.; ELTZ, R. D. Megabacteriose em emas (Rhea americana) no Estado do Rio Grande do Sul, Brasil. Ciência Rural, v. 34, n. 3, p. 959-960, 2004.

SON, T. T.; WILSON, G. H.; KENNETH, S. L. Clinical na Pathological Features of Megabacteriosis (Macrorhabdus ornithogaster) in Birds. Veterinary Clinical Pathology Clerkship Program. (2004). <Disponível em: www.vet.uga.du/vpp/clerk/Son/index.php>. <Acesso em: 01/12/2010>.

TOMASZEWSKY, E. K.; LOGAN, K. S.; SNOWDEN, K. F.; KURTZMAN, C. P.; PHALEN, D. N. Phylogenetic analysis identifies the 'megabacteria' of birds as a novel the anamorphic ascomyvetous yeast, Macrorhabdus ornithogaster gen. nov., sp. International Journal of Systematic and Evolutionary Microbiology, v. 53, p. 1201-1205, 2003.

WERTHER, K.; SCHOCKEN-ITURRINO, R. P.; VERONA, C. E. S.; BARROS, L. S. S. Megabacteriosis occurrence in budgerigars, canaries and lovebirds in Ribeirão Preto region São Paulo State - Brazil. Revista Brasileira de Ciência Avícola, Campinas, v. 2, n. 2, p. 183-187, 2000. 